\title{
Computer simulation of hippocampal place cells
}

\author{
PATRICIA E. SHARP \\ Yale University, New Haven, Connecticut
}

\begin{abstract}
Hippocampal pyramidal cells show location-specific firing as animals navigate through an environment. It has been suggested that this firing could result from the "local view" available in a cell's field. Hippocampal damage results in learning deficits on a wide variety of tasks. This, along with the fact that an associative form of plasticity has been discovered in the hippocampus has led to the idea that this structure might serve as a distributed, associative-matrix memory device. Here, these ideas are combined in a model in which pyramidal cells are the output layer of a competitive-learning, pattern-classification device. The inputs are patterns of environmental stimuli as viewed by a computerized "rat" from various locations within a simulated environment. These patterns are "classified" on the basis of their similarity. Since views available from contiguous regions of space are similar, single cells come to fire in a circumscribed region (place field). Firing-rate maps for these theoretical units show place fields remarkably similar to those of actual place cells. Also, they show remarkably similar behavior to that of real cells when tested under some of the probe conditions similar to those which have been used for actual cells.
\end{abstract}

Hippocampal pyramidal cells show location-specific firing as animals navigate through an environment (O'Keefe, 1976; O'Keefe \& Dostrovsky, 1971). These place fields are thought to be important in the role played by the hippocampus in spatial learning. It has been suggested that place fields may result from the "local view" available to an animal in the location of the cell's field (McNaughton, 1989; Zipser, 1985). Thus, it may be that sensory-driven activity of cells in the areas of association cortex known to project to the hippocampal formation causes particular hippocampal pyramidal cells to fire when particular conjunctions of stimuli are present. Simple neural-net models demonstrating the plausibility of this suggestion have been presented (Sharp, 1989; Skaggs \& McNaughton, 1989).

In addition to its clearly established role in spatial learning (Jarrard, 1983; Morris, Garrud, Rawlins, \& O'Keefe, 1982; O'Keefe \& Nadel, 1978; O'Keefe, Nadel, Keightly, \& Kill, 1975; Olton, Walker, \& Gage, 1978; Sutherland, Whishaw, \& Kolb, 1983), it is clear that the hippocampus plays a role in learning in many other tasks as well. Indeed, hippocampal damage in humans causes global deficits in memory for events and places (Corkin, 1984; Milner, Corkin, \& Teuber, 1968; Scoville \& Milner, 1957).

Insight into how the hippocampus may accomplish information storage in spatial and other tasks has come from physiological studies. Specifically, a long-lasting form of electrically induced synaptic plasticity, known as longterm potentiation or long-term enhancement, has been discovered here (Bliss \& Gardner-Medwin, 1973; Bliss \& Lomo, 1973), and has been shown to have the formal

This work was partially supported by NINCDS Grant NS 08408 to the author. Correspondence should be addressed to Patricia E. Sharp, Department of Psychology, P.O. Box 11A, Yale University, New Haven, CT 06520. properties outlined by Hebb (1949) in his theoretical mechanism of associative information storage in the nervous system (Barrionuevo \& Brown, 1983; Kelso \& Brown, 1986; Levy \& Steward, 1979; McNaughton, 1983; McNaughton \& Barnes, 1977; McNaughton, Douglas, \& Goddard, 1978). Apparently similar types of plasticity have also been induced by environmental, rather than electrical stimulation, suggesting that this plasticity may occur under natural learning conditions as well (Green, McNaughton, \& Barnes, 1990; Ruthrich, Matthies, \& Ott, 1982; Sharp, Barnes, \& McNaughton, 1987; Sharp, McNaughton, \& Barnes, 1985, 1989; Skelton, Scarth, Wilkie, Miller, \& Philips, 1987; Weisz, 1982).

These facts, along with certain salient anatomical features of the hippocampus such as its high divergence and convergence ratios (see Rolls, 1989; for review), have contributed to the general theoretical view that the hippocampus serves as a distributed, associative-matrix memory device for a wide variety of information (Marr, 1971; McNaughton, 1989; McNaughton \& Morris, 1987; Rolls, 1989).

The present model combines the idea that place fields result from "local views" with the idea that the hippocampus contains Hebb-like synaptic mechanisms that store patterns of sensory input, in order to model the activity of hippocampal place cells. For this, a simple neural network model, incorporating Hebb-like synaptic mechanisms, is used, with the hippocampal pyramidal cells being viewed as elements in the final, output layer of the device. The input layer consists of "neocortical cells" that have sensory responses to the elements of the patterns of stimuli available to the animal's sensory receptors from each of the various locations within an environment. Simulated rats run "sessions" in which they navigate through an environment, thus exposing themselves to various local views within it. 

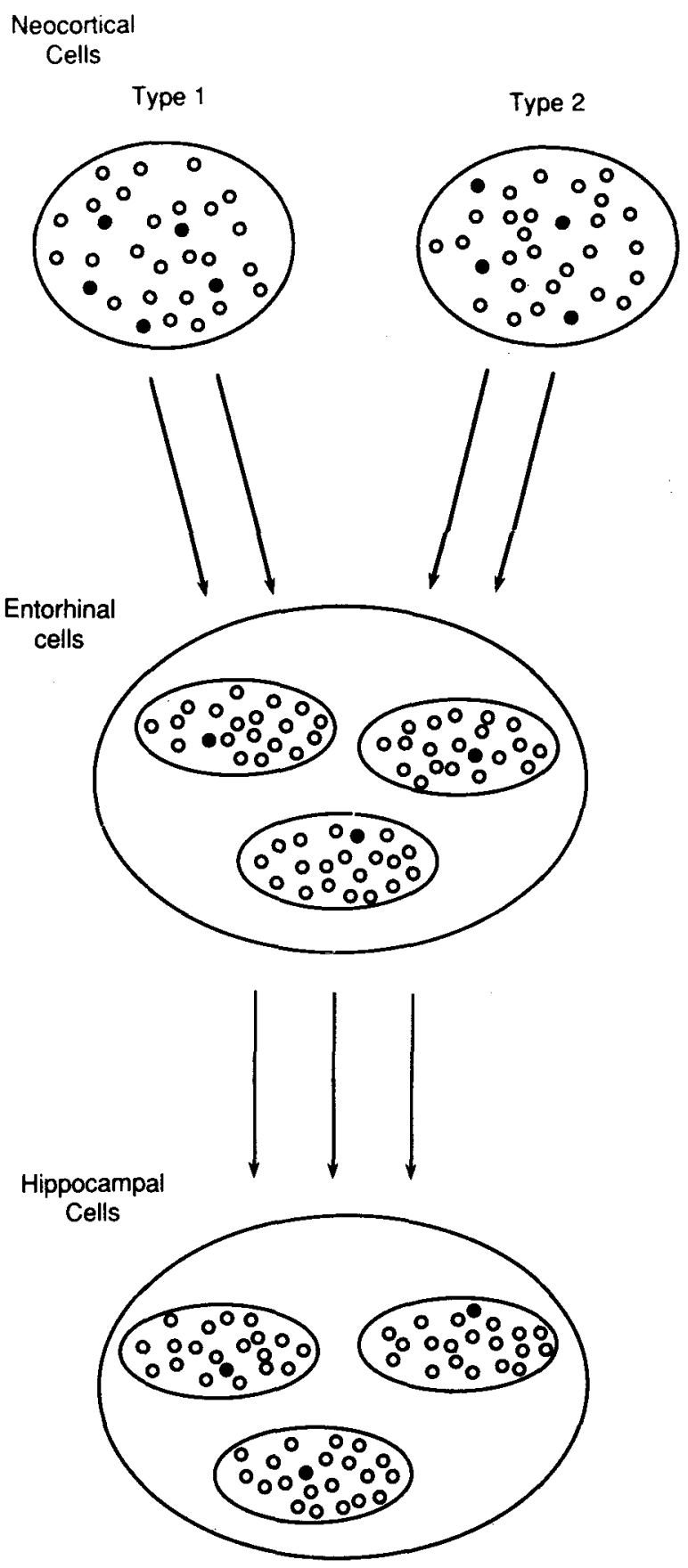

Figure 1. Schematic representation of the competitive-learning pattern-classification device, adapted from Rumelhart and Zipser (1986), used to simulate hippocampal cells. Each cell in a given layer makes a Hebb-like synaptic contact with every cell in the next lower layer. The strengths of these synaptic connections are initially randomized. Cells in the lower two layers are grouped into winner-takeall clusters, so that only the cell that receives the most synaptic input on any one occasion will fire. Cells in the first (neocortical) layer are sensory cells, which fire when a stimulus to which they are "responsive" is within range. See text for details.
The particular type of neural-network device used here is a competitive-learning, pattern-classification device (Grossberg, 1976a, 1976b; Rumelhart \& Zipser, 1986; Von der Malsburg, 1973). Such a device consists of two or more layers of neuron-like elements, with each element of a given layer being connected to each element of the next lower layer through a Hebb-like synapse (see Figure 1). Such devices come to classify and store input patterns on the basis of their similarity to one another, so that patterns that are similar (i.e., that share many elements in common) are likely to be represented by (to activate) the same output layer units.

This type of device was thought to be appropriate for the simulation of place cells, since places consist of patterns of simultaneously available stimuli. The configurations available from contiguous locations are similar, and so might be placed into the same class by such a device, thus resulting in a firing pattern in which a given cell fires over a circumscribed region of space (a place field). Also, these devices show pattern completion, as do hippocampal place cells (O'Keefe \& Conway, 1978), and their output patterns are dependent on plastic properties of the system, as are those of place cells (Bostock, Muller, \& Kubie, 1991; Sharp, Kubie, \& Muller, 1990). In addition, the model is compatible with a generalized view of place cells as responding to a wide variety of relations between stimuli, other than just those which define a particular place (Eichenbaum \& Cohen, 1988). Finally, the model is compatible with the idea, based on deficits resulting from hippocampal formation damage, that the hippocampus is involved in storing configural representations (Sutherland \& Rudy, 1989).

Thus, in this model, place-cell firing itself is seen as a stored configural representation of environmental stimuli. Place fields, then, are viewed as one example of the type of stored representation postulated to exist in the hippocampus. These representations can be reinstated even in the face of modest environmental changes. They presumably provide the rest of the brain with configural representations that can be used in other sorts of informationprocessing and -storage tasks.

\section{THE MODEL}

The competitive-learning pattern-classification device used here was taken, almost without modification, from that of Rumelhart and Zipser (1986), to whom the reader is referred for a theoretical discussion of the device itself. Units in the first layer (see Figure 1) are conceived of as sensory cells, each of which is activated by particular "environmental stimuli" to which it happens to be "responsive." One modification to the Rumelhart and Zipser model is that here, this input layer is divided into two types of units with somewhat different response properties, as described below. Subsequent layers are divided into winner-take-all clusters, which means that only the one cell within each cluster that receives the largest input on 
any one occasion will fire. Every cell in a given layer receives inputs from every cell in the layer above, with the strength of the connection between any two cells being randomized initially. All weights are positive, and the sum of these connective strengths is normalized over each postsynaptic cell. Determination of which cell within a cluster receives the largest input is accomplished through first multiplying, for each cell, the activity level on each of the input lines (which is either 1 , if the line is active, or 0 , if it is inactive) by the strength of its connection with that line (i.e., the synaptic strength), and then summing over these products. Thus, the inner product of the input (or presynaptic) activity vector and the synaptic weight vector is calculated. The cell, within each cluster, that has the largest inner product fires, while the others remain silent. This winner-take-all clustering of units is a simple way of simulating both feedforward and feedback inhibition provided in the brain by inhibitory interneurons. Such inhibitory influences are thought to act to keep the global activity level in a region constant, even though the number of active cells in the input layer may vary dramatically, as a function of external stimulus input (Marr, 1969). Interneurons thought to be capable of providing both feedforward and feedback inhibition are found throughout the hippocampus (Andersen, Eccles, \& Loyning, 1964; Lorente de No, 1934; Ramon y Cajal, 1911; see McNaughton \& Morris, 1987, for review).

Cells "learn" only on occasions on which they fire. When this happens, each synaptic connection first loses a portion of its strength, and then the sum of this decrease in strength is redistributed among the connections that have active input lines. In this way, all connections that were active when the cell fired become strengthened. (It should be noted that because of the way that synaptic strength is redistributed on each occasion, the total amount of synaptic strength for any one postsynaptic cell remains fixed, and identical to that of all other cells; see Grossberg, 1976a, 1976b, for a discussion.) This means that on subsequent occasions, that unit is more likely to fire to that pattern, or to any pattern similar to (having a large proportion of elements in common with) that one.

In this way, each layer comes to classify the patterns from the layer just above it in terms of their similarity. The system can also classify novel patterns, or impoverished versions of already learned ones.

\section{APPLICATION OF THE PATTERN-CLASSIFICATION DEVICE TO THE HIPPOCAMPAL FORMATION}

In order to apply this device to place-field generation, it was first necessary to decide how to set up activity patterns in the initial, sensory cell layer. Several considerations went into this decision. First, it was important that the sensory cells have "response properties" that resembled, as closely as possible, those of cells in areas of the neocortex that project to the hippocampal formation. It is known that the entorhinal cortex, either directly or in- directly, receives inputs from broad areas of polysensory association cortex (Insausti, Amaral, \& Cowan, 1987; Van Hoesen \& Pandya, 1975; Van Hoesen, Pandya, \& Butters, 1975). Although little is known about the physiology of most of these areas, available data suggest that these cells have optimal stimuli that are complex patterns or objects, that they have broad receptive fields, and, in cases in which it has been tested, that they have object constancy (Baylis, Rolls, \& Leonard, 1987; Miyashita \& Chang, 1988). Second, it was thought important to use a simulation of an environment that has actually been used to study hippocampal place cells, so that the modeled results could be compared directly to real place-cell data. Finally, it is known that the output of pattern-classification devices is affected by the order and frequency with which each of the patterns is presented (see Rumelhart \& Zipser, 1986 , for a theoretical discussion). Because of this, it was thought that the series of "local views" of the environment should be presented to the system in the most realistic way possible.

To accommodate each of these considerations, the simulated environment shown in Figure 2 was constructed. The environment itself consists of a circular array of eight point stimuli. These are meant to simulate the cylindrical apparatus which has been used in numerous studies of place cells (e.g., Muller \& Kubie, 1987; Muller, Kubie, \& Ranck, 1987). In this recording paradigm, rats are placed into a uniformly painted, gray, cylindrical apparatus ( $76 \mathrm{~cm}$ in diameter), which has one white card that occupies $100^{\circ}$ of arc and extends from the floor to the ceiling of the apparatus. The animals are food-deprived,

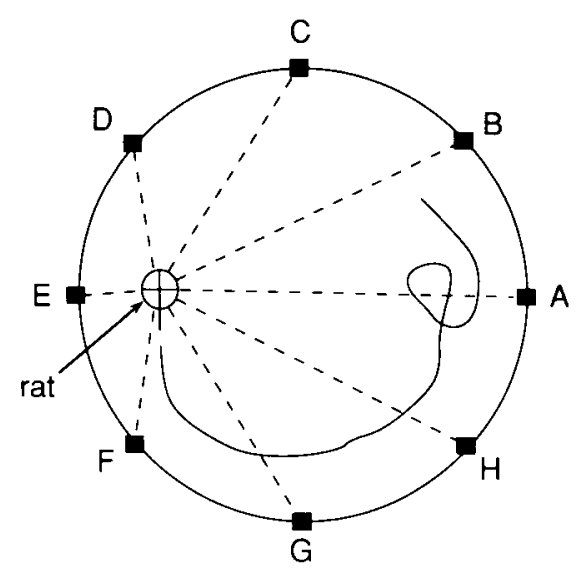

Figure 2. Schematic representation of the simulated environment and rat. The circular array of eight point stimuli provides a simulation of the cylindrical recording apparatus used to record from real hippocampal cells (e.g., Muller \& Kubie, 1987; Muller, Kubie, \& Ranck, 1987). The simulated rat runs "sessions" in this environment, consisting of constant locomotion in patterns made to replicate those of actual rats as closely as possible. At each small quantum "step" that the animal takes, calculations are made of the distance and angle of each of the point stimuli in relation to the rat's current head position. The results of these calculations are used to set up firing patterns in the first (neocortical) layer of cells in the pattern-classification device shown in Figure 1. 
and small food pellets are thrown into the apparatus at pseudorandom locations throughout recording sessions. Rats in this situation come to generate nearly continuous patterns of locomotion, in which they zigzag through the apparatus in a wide variety of apparently unpredictable, but characteristic, patterns.

The use here of the eight point stimuli (Figure 2) to simulate this environment was based on the assumption that each section of the cylinder presents a different stimulus for cells in association cortex. Thus, Stimulus A could be thought of as representing a section of the cylinder that is covered by the uniform, white card, while Stimulus B would contain the edge of the card against the gray wall, and so forth.

The computerized "rat" that ran "sessions" in this simulated environment is also shown in Figure 2. During such sessions, the "rat" takes small, quantum jumps (each covering a distance corresponding to $7 \%$ of the diameter of the cylindrical enclosure). The overall pattern of these steps was made to simulate, as closely as possible, the actual patterns of trajectories taken by real rats in this situation. The size of each quantum leap was made to approximate the average distance (in relation to the size of the environment) traveled by an actual rat in $1 / 8$ of a second, and the reason for choosing this distance is explained below.

At each step that the "animal" takes, calculations are made of the angle and distance of each of the eight stimuli with respect to the "animal's" current head position. These calculations are used to set up activity in the input layer of neocortical cells. Each cell in this layer has a 0.17 probability of being "responsive"' to each of the environmental stimuli. Random determination of which stimuli each of the cells is responsive to is made at the beginning of the simulations for any one "rat." There are two types of these cells. Type 1 cells fire whenever the "rat" is within a certain range of a stimulus to which it is responsive. Each such cell has a characteristic range that varies, across cells, between 15 and 40 "'cm," in relation to the simulated 76-cm-diam cylinder. Type 2 cells are similar to Type 1 cells in that they fire only when the "rat" is within a given, characteristic distance of a stimulus to which they are responsive. They have the additional requirement, however, that the stimulus be within a certain range of angle to the "animal's" head. Thus, these cells have a receptive field that covers a given portion of the angular distance around the "animal's" head. The size of this receptive field varies between $80^{\circ}$ and $170^{\circ}$ across cells. This has the effect that, for Type 2 cells, responses are dependent not only on the "animal's" current location, but also on the direction in which it is facing.

There are 60 Layer 1 cells, with 30 in the Type 1 and 30 in the Type 2 categories. It should be noted that there is no limit on the number of Layer 1 cells that may fire on any one occasion, which is not the case with the cells in subsequent layers.
Once firing is established in the input layer, the pattern projects through the middle layer, which has been designated here as the entorhinal cortex. This is in keeping with anatomical studies in which it has been shown that association areas of neocortex project to the entorhinal cortex - which in turn provides the hippocampus with its main source of cortical afferent input. There are 60 entorhinal cells, divided into 3 clusters. As described above, 1 cell in each of these clusters is selected to fire on the basis of the strength of its connections with currently active cells. The cell that fires on any one occasion has its synaptic weights changed in accordance with the Hebb-like rules described above.

Finally, the pattern of activity from the entorhinal cortex (consisting of 3 active cells at any one time) projects onto the hippocampal layer. Here, there is only one cluster of 20 cells. The rules for choosing which cell is active and for synaptic plasticity are identical to those already described. One slight modification in this layer, however, is that an active cell (i.e., a cell that has won the competition) may be in one of two states. If it has an inner product (of the presynaptic activity vector and the synaptic weight vector) of 0.25 or less, it fires a single spike. If, however, its inner product is above this level, it fires a complex spike (Ranck, 1973), which consists of either 3 or 5 action potentials, depending on how much above the 0.25 level it is. Thus, the momentary rate for these cells is dependent on the degree of similarity between the incoming activity pattern and the synaptic weight vector.

"Animals" run 16-min "sessions" of constant navigation, with the input patterns generated at each step processed through the three layers in the manner described. Note that since the "rats" take steps at a simulated rate of $8 \mathrm{~Hz}$, this means that there are a total of 7,680 inputs presented to the model for each such session.

Over the course of the sessions, a record is kept of which cells fire during each step the "animal" takes. Afterwards, a firing rate map can be constructed in which the rate of firing as a function of location is calculated for an array of pixels covering the apparatus floor. This manner of recording and displaying firing rate as a function of location is directly analogous to that used for real hippocampal cells, in which cell activity and the "animal's" momentary location are recorded for the purpose of firing-rate map construction (Muller \& Kubie, 1987; Muller et al., 1987).

Finally, it is necessary to comment on why, as described above, the simulated "steps" taken by the "rat" were made to correspond in size to the relative distance traveled by a real rat in about $1 / 8$ of a second. (Note that this means that the input patterns presented to the pattern-classification device are delivered at a simulated rate of about $8 \mathrm{~Hz}$.) The reason for choosing this rate is that it corresponds to the approximate frequency of the theta EEG pattern in the hippocampus, which is present whenever rats engage in locomotor behavior (Vanderwolf, 1969). It has 
been shown that hippocampal pyramidal cells fire at theta frequency when theta is present (Fox, Wolfson, \& Ranck, 1986). Thus, it seemed reasonable to guess that, in real rats, local views of the environment are processed in the hippocampus at a rate of about $8 \mathrm{~Hz}$.

\section{RESULTS}

Typical firing-rate maps for a set of hippocampal cells from one simulated hippocampus, from one 16-min session that was conducted after a total of $64 \mathrm{~min}$ of simulated session time, are shown in Figure 3A. Note that only the maps for the cells that were active in this environment are shown; most of the 20 hippocampal cells were silent. For comparison, a set of firing-rate maps from actual hippocampal cells is also displayed in Figure 3B. It can be seen that the simulated cells display a locationspecific pattern remarkably similar to that of the real hippocampal cells. Both cell types display patterns that tend to occupy from $20 \%$ to $50 \%$ of the area of the cylinder, tend to have a shape that conforms to the contours of the cylinder, and are shaped like a bullseye, with the highest firing in the middle of the field. These basic characteristics were noted by Muller et al. (1987). Thus, the basic phenomenon of place-cell firing is generated here.
It should be noted that the properties of place cells observed here are dependent on the plastic properties of the synapses in the system. When simulations are conducted in which the changes in synaptic weight are turned off, the firing patterns are patchy, and tend to be scattered throughout the cylinder, with no clear field.

\section{FURTHER COMPARISONS OF ACTUAL AND SIMULATED HIPPOCAMPAL CELLS}

\section{Fields Are Stable Over Time}

One interesting aspect of place-cell firing is that, although it has long been suspected that the firing properties are dependent on experience (plasticity), existing evidence has suggested that fields are present from the first instant that an animal is placed into an environment (Hill, 1978), and that, in any one environment, these fields remain stable over long periods of time (Best \& Thompson, 1989; Muller et al., 1987). This is also true for the cells modeled here. Firing-rate maps from one cell are shown in Figure 4, for the 1st, 5th, and 10th consecutive 16min sessions. The field is similar in size, shape, and location across the sessions. To obtain a quantitative measure of the similarity between these firing-rate maps, a pixel by pixel correlation (Muller \& Kubie, 1987) was
A

SIMULATED PLACE CELLS
B

\section{ACTUAL PLACE CELLS}
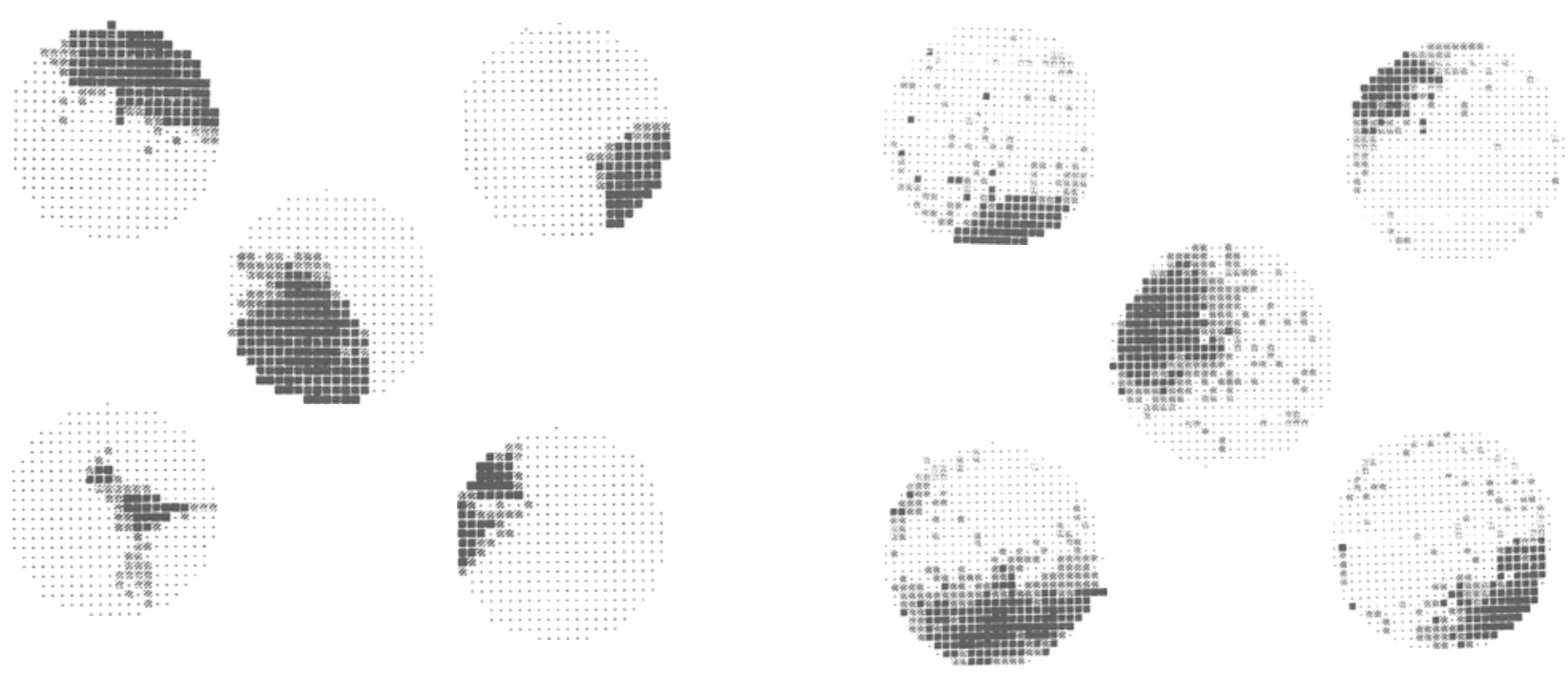

Figure 3. (A) Firing-rate maps for a typical set of simulated "hippocampal cells" from one "rat." For each map, the total number of times that the given cell fired in each location during the simulated 16-min session was divided by the total amount of time that the "rat" spent in that location. For the purpose of display, the area of the cylinder floor was then divided into a set of pixels, with the average rate in each pixel being indicated by the darkness of the shading within it. Darker colors correspond to higher rates, and the color scale is determined in a relative fashion. (B) Firing-rate maps for typical examples of actual hippocampal pyramidal cells, recorded from rats performing a pellet-chasing task during a 16-min session in a cylindrical apparatus. Recordings of the cellular activity, as well as the animal's momentary location, were made throughout the session, and firing-rate maps were constructed in a manner analogous to that described for Figure 3A. It can be seen that these rate maps for the simulated and actual cells are remarkably similar. 
SESSION 1 SESSION 5

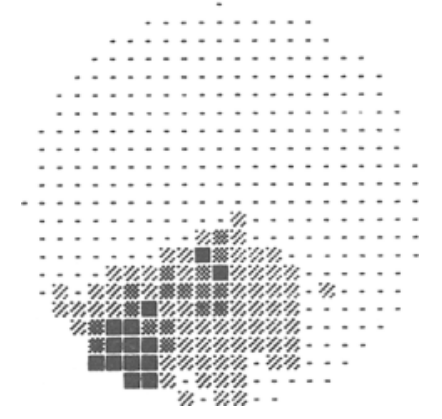

SESSION 10

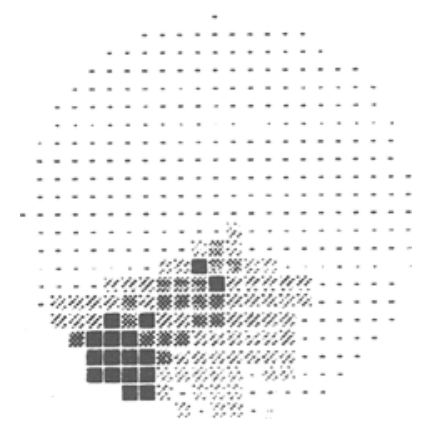

Figure 4. Firing-rate maps (as in Figure 3A) for a simulated cell from a different "rat" during the 1st, 5th, and 10th 16-min sessions. The field was similar in size and shape across these sessions, illustrating the stability of these fields over time.

conducted. The $R$ for the correlation of the map from Session 1 with that from Session 5 was 0.72 , whereas that for Sessions 5 and 10 was 0.98 . The difference in these two scores suggests that the field was not exactly in its final form during Session 1, suggesting that there is at least some experience-dependent change over the first minutes in an environment. The value for Sessions 5 and 10 is somewhat higher than that obtained using a similar measure for actual hippocampal cells over repeated sessions (Muller \& Kubie, 1987; Muller et al., 1987), suggesting that there are fewer sources of variance influencing the firing of modeled cells than there are in actual cells, as would be expected.

\section{Fields Persist Even When Some of the Controlling Cues are Removed}

Early work with a set of four experimenter-controlled cues known to jointly control the location of fields showed that removal of any subset of the cues left the fields of many cells intact (O'Keefe \& Conway, 1978). Firing-rate maps for examples of modeled cells in which subsets of two stimuli were removed are shown in Figure 5. It can be seen that the fields were quite similar in size and shape even after removal of one quarter of the controlling cues.

\section{Fields Have Directional Correlates Under Some Conditions}

When place cells have been studied under conditions in which rats are performing on an eight-arm maze, their firing rate has been shown to be dependent on the direction in which the animal is facing (McNaughton, Barnes, \& O'Keefe, 1983). Results obtained from animals performing in the cylindrical apparatus being simulated here, however, usually show no detectable variation as a function of direction (Bostock, Taube, \& Muller, 1988).

It was reasoned here that these results might be due to the differences in trajectories taken by animals in the two circumstances. In the eight-arm maze, trajectories are tightly restricted to either inward or outward paths on the maze arms, whereas in the cylinder, animals move through any one location in a variety of patterns, and thus they view the same location from many different directions.

In terms of a pattern-classification device, a system in which the same cell fires to the "local view" available from different directions within the same location has placed those views into the same class. The likelihood that any two patterns will be placed into the same class depends on a number of factors. One obvious major factor is the similarity of the two views-that is, the number of elements that they have in common. For the present model, any two views from the same location can be expected to have all of their Type 1 neocortical cell inputs in common, since these cells respond only as a function of distance from environmental stimuli. They would not, however, be expected to have many Type 2 units in common. Another factor influencing the likelihood that any two patterns will be placed in the same class has to do with the structure of the entire set of patterns that are presented. In particular, this likelihood also depends on how many patterns intermediate between the two are experienced (where intermediate means that the pattern has more stimulus elements in common with both the patterns in question than they have with each other). Thus, if the two views available when the animal is facing two opposite directions in a given location are experienced in addition to other views available from intermediate directions within that location, the likelihood that those two opposite views will be placed into the same category is increased. This is because, in this case, the two opposite views are part of a "cluster" (a set of stimulus patterns that have relatively large subsets of their elements in common) of similar patterns in the input space (see Rumelhart \& Zipser, 1986). Alternatively, if no views of intermediate directions are available, the views from the local 

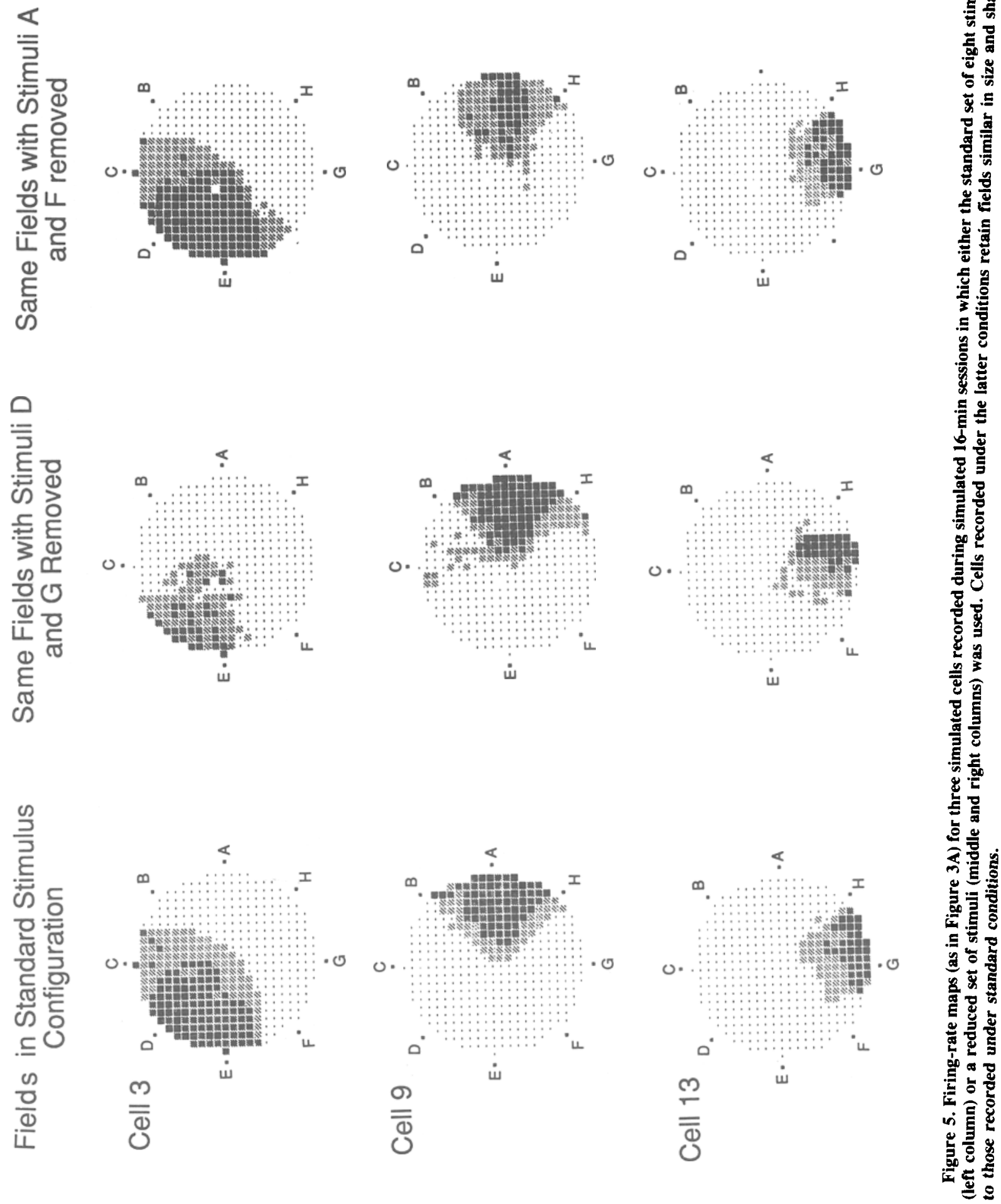


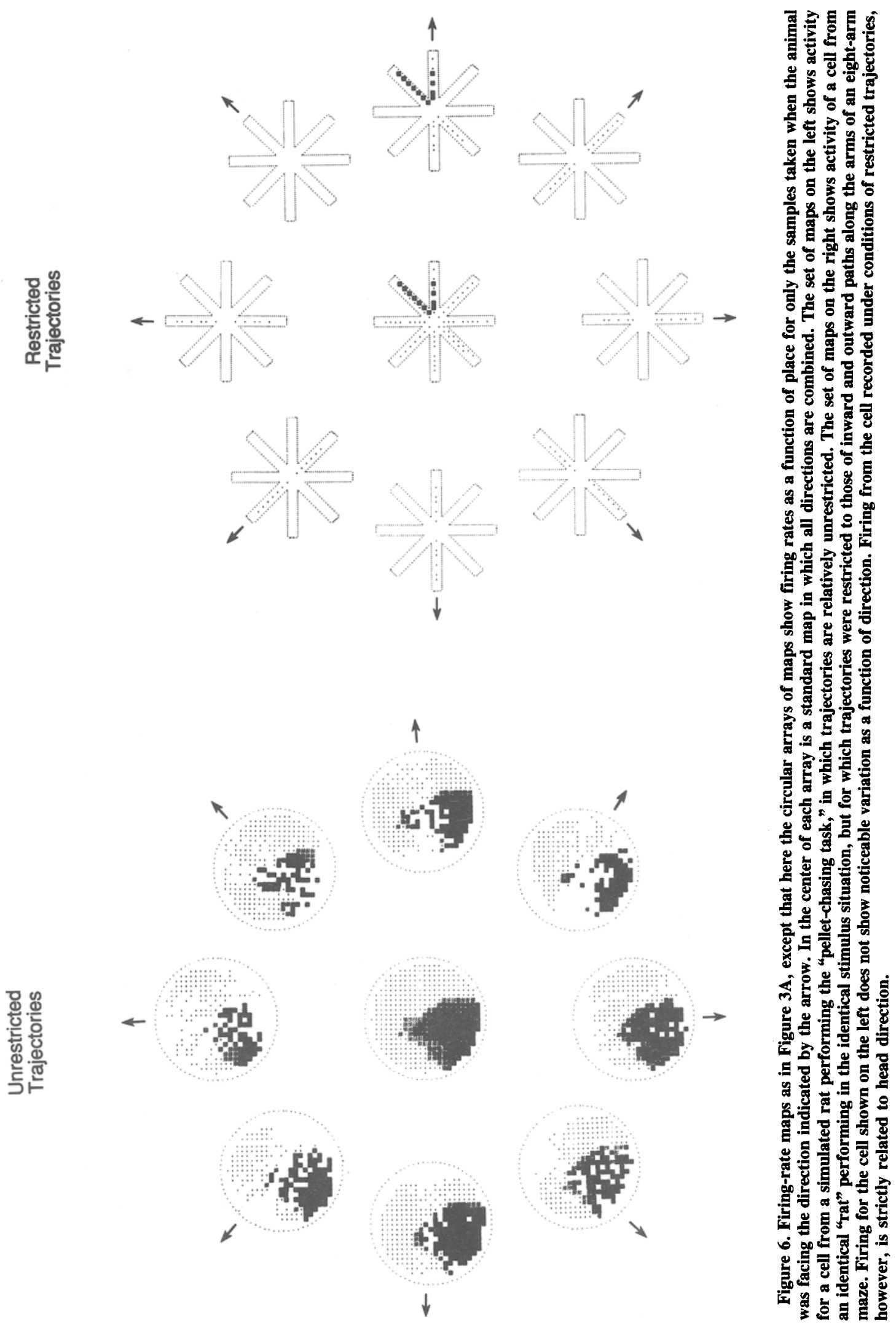


region for each of the two directions may each form their own "cluster" in the input space, and so they would be less likely to be placed in the same category.

The firing patterns of the two cells in Figure 6 are taken from two identical "rats," one of which had been trained in the simulated cylindrical apparatus already described (top), and the other of which had been trained in a simulated eight-arm maze (bottom). All conditions of the model and environmental stimuli were identical for the two "rats," except that for the "rat" that ran on the eightarm maze, trajectories were restricted to inward and outward paths on the arms. The circular arrays of firing-rate maps show firing rate as a function of place for only the samples taken when the "animal" was facing in the direction indicated by the arrow. In the center is a standard map in which all directions are combined. It can be seen that, in the cylindrical apparatus, the firing pattern is not noticeably affected by the direction in which the "animal" is facing. In the maze, however, the cell fires only when the "animal" is facing one of the possible directions. Of the total set of cells tested, only 3 out of 19 were directional (as assessed visually) in the simulated cylindrical apparatus, whereas 14 out of 20 were directional in the maze.

\section{Increases in the Size of the Testing Environment Lead to Increases in the Size of the Fields}

Original studies of place cells in the cylindrical apparatus showed that when a given cell was tested in a version of the environment that was identical to the standard in all ways, except that it had been increased in size by a factor of two, fields often showed a similar increase in size, while maintaining the same relative position and shape (Muller \& Kubie, 1987).

To test the output of the present model against these results, sessions were conducted in which the same "rat" was exposed to both the standard stimulus array and one in which the diameter of the circular array had been doubled. All other parametric settings of the model were left
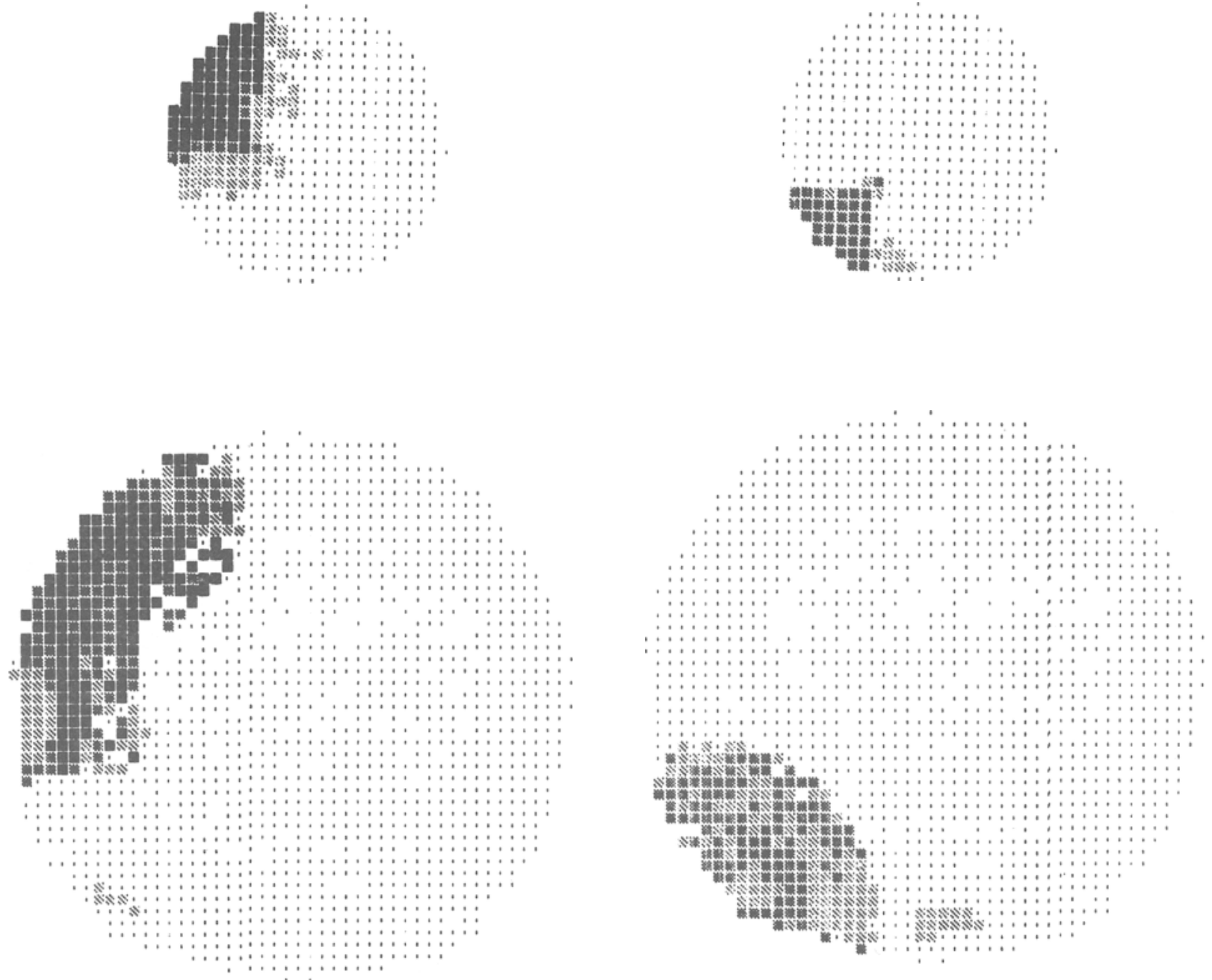

Figure 7. Firing-rate maps (as in Figure 3A) for two different cells in the standard array of stimuli (top) as well as an expanded version of the same array (bottom). Fields in the two stimulus configurations are similar in size and location, in relation to their respective stimulus arrays. Thus, the fields are "scaled up" in the large cylinder. 
the same. Of the total of 24 cells tested, 13 had fields in both the small and the large cylinder. Of these, 10 had fields that were related in the two cylinders, in that the field in the large cylinder was similar in shape and location to that in the small cylinder. In most of these cases, the field in the large cylinder was a larger version of the small cylinder field. Two examples of cells of this type are shown in Figure 7. The remaining 11 of the total 24 cells had a field in only one environment. These results are remarkably similar to those of Muller and Kubie (1987) for actual hippocampal cells studied in this paradigm. Of the 22 cells that they tested, 13 had fields in both cylinders, and these were similar to each other in 9 of these cases. Nine cells had fields in only one of the environments.

\section{LIMITATIONS OF THE MODEL}

One set of phenomena not addressed by the current model consists of those in which all environmental cues that are controlling factors for a place field are removed. Specifically, experiments have been conducted in which place fields are studied in the presence of a set of experimenter-defined cues, which can be demonstrated to jointly have complete control over the location of a cell's field. It has been shown that, under these conditions, when all the cues are made to disappear, either through removal or through extinguishing the room lights, fields remain intact, provided that the animal has been introduced into the environment prior to cue removal (Jones Leonard, McNaughton, \& Barnes, 1985; O'Keefe, 1976; O'Keefe \& Speakman, 1987; Quirk, Muller, Kubie, \& Ranck, 1987). If, however, the animal is brought into the environment in the dark or after cue removal, the cell shows some unpredictable firing pattern.

It has been suggested that this ability to maintain the same firing field in the absence of all controlling cues must be dependent on some sort of information about ongoing motor activity, along with stored information about the environment and the sensory consequences of particular movements within it (McNaughton, 1989; McNaughton \& Morris, 1987).

The present model provides no inputs related to the simulated motor patterns, and so it is incapable of generating such results. Further developments could, in principle, however, successfully incorporate such information.

\section{CONCLUSIONS}

In the simulation presented here, many of the basic properties of hippocampal place cells have been replicated through the use of a very simple model in which simulated sensory responses to the various local views available in an environment serve as inputs to a competitivelearning, pattern-classification device. The various patterns of sensory responses to environmental stimuli are classified according to their similarity to one another. Since contiguous regions of space offer similar views, cells come to respond within circumscribed regions, or place fields.

Interpreted in the most general sense, the current model simply provides a demonstration that a neural-net device, when implemented with some reasonable set of assumptions about the hippocampal formation, can replicate certain place-cell-like phenomena. Thus, it is similar to any number of neural-net simulations that have appeared over the past several years, and which have proven able to simulate a wide variety of brain-like activity patterns. The apparent ease with which such devices can be constructed has fueled understandable enthusiasm about them, and would seem to lend support to the idea that the general principles utilized by these devices (i.e., parallel distributed processing and Hebb-like synaptic plasticity) are likely to be similar to processes actually found in the brain.

If any particular model of a given brain region is to be scientifically useful beyond this general level of demonstration, however, it must make clear its own specific, testable claims about how the region performs a given information-processing task. Indeed, the very ease with which these devices can simulate neural behavior patterns makes it clear that any particular behavior could likely be generated by any number of such devices, each of which could possibly utilize very different assumptions about the structure of the modeled system and the nature of its inputs.

One difficulty involved in elucidating the specific claims of a model is to make clear which aspects of the model are meant to be fundamental (meaning that their violation would invalidate the model) and which are assumed to be trivial. Thus, in the present model, many decisions were made about details such as the number of cells in each layer, the number of cells within each "cluster," the exact nature of neocortical sensory responses, the number of environmental stimuli, and so forth. Obviously, many of these aspects of the model are either incorrect (e.g., the entorhinal cortex does not consist of just 60 cells) or, at best, oversimplifications. Decisions were made about them, and incorporated into a working model, in order to demonstrate that the assertions of the model could, at least in one case, actually lead to the replication of place-cell-like activity.

Other aspects of the model, however, are assumed to be central to the theoretical claim being made. The fundamental assertions of the present model are the following:

1. The hippocampal formation receives input from cells that individually respond to selective aspects or components of the overall environmental stimulus array. In any environment in which the place-cell phenomenon is actually exhibited, the pattern of activity in this input layer changes as a function of the animal's location, and it does so in such a way that, in general, the patterns in two contiguous regions are more similar than the patterns in two regions that are far apart.

Although other types of input may also be present (such as motor information), the input described above must play a large role, at least during the initial exposures to 
an environment, in determining which hippocampal cells fire.

2. The connectivity within the system shows high levels of both divergence and convergence.

3 . The system shows Hebb-like properties, so that conjunctive activity of a pre- and postsynaptic element causes an increase in the strength of the connection between them.

4. There is some kind of regulatory influence, such as that provided by inhibitory interneurons, on the overall activity level of the system, so that only the cells that receive the most total synaptic input, relative to other cells in the area, will fire. Here, this inhibitory influence is modeled by the winner-take-all cluster organization within each layer.

Each of these four assumptions is compatible with what is known about hippocampal anatomy and physiology, and the justification for each has been discussed individually above.

A set of predictions can be generated from this basic set of properties:

1. The likelihood that any two locations will be represented by the same cell is influenced by the similarity (in terms of sensory qualities) of the two locations. Thus, for example, if animals were trained in an environment that included sets of identical "local views" at different locations, it would be expected that the probability of the same place-cell's firing in any two such locations would be greater than that for two locations that differed in their sensory qualities.

Sharp et al. (1990) have presented data compatible with this prediction, although they also emphasize that the model, in its current form, is incomplete. In this study, animals were trained to chase food pellets in the cylindrical apparatus described above. Lengthy initial training was conducted with a single white card on the wall. Once a cell with a place field was recorded in this apparatus, additional sessions were conducted in which a second, identical card was added $180^{\circ}$ away from the first. In this configuration, there are pairs of visually identical locations, $180^{\circ}$ apart. Most cells recorded under these conditions continued to show a single, asymmetrical firing pattern, so that the field appeared in only one of two identical locations within any one session, but varied between the two locations across sessions. This pattern indicates that immediately present visual stimuli are not the only inputs to the system, and it suggests that mnemonic influences (related to the initial, single-card training), in conjunction with motor information, may also be involved. (This point was raised above in the section on limitations of the model.) Further development of the model, incorporating movement-related information, will be tested against these results.

In contradistinction to the asymmetric pattern shown by most cells, however, a small subset of the cells recorded in the two-card configuration showed paired, symmetrical firing fields, so that they showed similar firing in the two visually identical locations. Thus, sensory similarity increased the likelihood of two areas' being represented by the same cell. It is postulated here that if the initial training were also conducted in the two-cue environment, this likelihood would be further increased, since mnemonically based representations of the singlecard environment would not be present.

2. The likelihood that two locations (or views within locations) with some sensory overlap would be represented by the same cell should be influenced by the amount of exposure that the animal has to the area between the two locations (assuming that this area is more similar sensorially to each of the two locations than they are to each other). The reasoning for this prediction is the same as that presented above, along with supportive data, in the section on directional correlates. Specifically, the likelihood that any two input patterns will be represented by the same cell is influenced by the extent to which they are part of the same "cluster" of patterns in the total set of input patterns. This, in turn, is related to the presence of views intermediate to the two. Studies are currently being designed to test the likelihood that the same place cell will fire in two different locations as a function of whether the animal is allowed to travel through a direct trajectory between them.

3. Place fields should be resistant to the removal of small subsets of stimuli, and the particular stimuli chosen should not make a difference. Data have already demonstrated this property (see, e.g., Muller \& Kubie, 1987; O'Keefe \& Conway, 1978).

Substantial verification of each of these three predictions should provide evidence that much of the determination of place-cell firing patterns takes place through a process in which the hippocampal formation acts as a patternclassification device for sensory inputs.

This, in turn, suggests that much of the role played by the hippocampus in spatial and other kinds of learning is to provide for the formation and cued recall of representations of complex stimulus arrays, such as those which uniquely identify events and places.

\section{REFERENCES}

ANdersen, P. , EcCles, J. S. , LoYNing, Y. (1964). Location of postsynaptic inhibitory synapses of hippocampal pyramids. Joumal of Neurophysiology, 27, 592-607.

Barrionuevo, G., \& Brown, T. H. (1983). Associative long-term potentiation in hippocampal slices. Proceedings of the National Academy of Sciences, 80, 7347-7351.

Baylis, G. C., Rolls, E. T., Leonard, C. M. (1987). Functional subdivisions of the temporal lobe neocortex. Joumal of Neuroscience, 7, 330-342.

Best, P. J., Thompson, L. T. (1989). Persistence, reticence, and opportunism of place-field activity in hippocampal neurons. Psychobiology, 17, 230-235.

Buss, T. V. P., \& GARDNER-Medwin, A. R. (1973). Long-lasting potentiation of synaptic transmission in the dentate area of the unanesthetized rabbit following stimulation of the perforant path. Joumal of Physiology, 232, 357-374.

Bliss, T. V. P., \& Lomo, T. (1973). Long-lasting potentiation of synaptic transmission in the dentate area of the anesthetized rabbit following stimulation of the perforant path. Journal of Physiology, 232, 331-356.

Bostock, E., Muller, R. U., \& Kubie, J. L. (1991). Experience- 
dependent modification of hippocampal place cells. Hippocampus, $\mathbf{1}$, 193-206.

Bostock, E., TAUbe, J., \& MULLER, R. U. (1988). The effects of head orientation on the firing of hippocampal place cells. Society for Neuroscience Abstracts, 14, 127.

CorkIN, S. (1984). Lasting consequences of temporal lobectomy: Clinical course and experimental findings in H.M. Seminars in Neurology, 4, 249-259.

Eichenbaum, H., Cohen, N. J. (1988). Representation in the hippocampus: What do hippocampal neurons code? Trends in Neurosciences, 11, 244-248.

Fox, S. E., Wolfson, S., \& RANCK, J. B., JR. (1986). Hippocampal theta rhythm and the firing of neurons in walking and urethane anesthetized rats. Experimental Brain Research, 62, 495-508.

Green, E. J., McNaughton, B. L., \& Barnes, C. A. (1990). Exploration dependent modulation of synaptic responses in fascia dentata: Dissociation of motor, EEG, and sensory factors and evidence for a synaptic efficacy change. Journal of Neuroscience, 10, 1455-1471.

GrossBerg, S. (1976a). Adaptive pattern classification and universal recoding: I. Parallel development and recoding of neural feature detectors. Biological Cybernetics, 23, 121-134.

Grossberg, S. (1976b). On the development of feature detectors in the visual cortex with applications to learning and reaction-diffusion systems. Biological Cybernetics, 21, 145-159.

HeBB, D. O. (1949). The organization of behavior. New York: Wiley.

HiLL, A. J. (1978). First occurrence of hippocampal spatial firing in a new environment. Experimental Neurology, 62, 282-297.

Insausti, R., Amaral, D. G., \& Cowan, W. M. (1987). The entorhinal cortex of the monkey: II. Cortical afferents. Journal of Comparative Neurology, 264, 356-395.

JARRARD, L. E. (1983). Selective hippocampal lesions and behavior: Effects of kainic acid lesions on performance of place and cue tasks. Behavioral Neuroscience, 97, 873-889.

Jones Leonard, B., McNaughton, B. L., \& Barnes, C. A. (1985). Long-term studies of place field interrelationships in dentate gyrus neurons. Society for Neuroscience Abstracts, 11, 1108.

Kelso, S. R., \& BRowN, T. H. (1986). Differential conditioning of associative synaptic enhancement in hippocampal brain slices. Science, 232, 85-87.

LEVY, W. B., STEWARD, O. (1979). Synapses as associative memory elements in the hippocampal formation. Brain Research, 175, 133-245.

LORENTE DE No, R. (1934). Studies on the structure of the cerebral cortex: II. Continuation of the study of the ammonic system. Zeitschrift für Psychologie und Neurologie, 46, 113-177.

MARR, D. (1969). A theory of cerebellar cortex. Journal of Physiology, 202, 437-470.

MARR, D. (1971). Simple memory: A theory for archicortex. Proceedings of the Royal Society: Series B, 262, 23-81.

McNaughton, B. L. (1983). Activity dependent modulation of hippocampal synaptic efficacy: Some implications for memory processes. In W. Seifert (Ed.), The neurobiology of the hippocampus (pp. 233251). London: Academic Press.

McNaughton, B. L. (1989). Neural mechanisms for spatial computation and information storage. In L. A. Nadel, P. Cooper, P. Culicover, \& R. Harnish (Eds.), Neural connections and mental computations (pp. 285-349). Cambridge, MA: MIT Press.

McNaughton, B. L., \& Barnes, C. A. (1977). Physiological identification and analysis of dentate granule cell responses to stimulation of the medial and lateral perforant pathways in the rat. Journal of Comparative Neurology, 175, 439-453.

McNaughton, B. L., Barnes, C. A., \& O'Keefe, J. (1983). The contribution of position, direction and velocity to single unit activity in the hippocampus of freely-moving rats. Experimental Brain Research, $52,41-49$.

McNaughton, B. L., Douglas, R. M., Goddard, G. V. (1978). Synaptic enhancement in the fascia dentata: Cooperativity among coactive afferents. Brain Research, 157, 277-293.

MCNAughton, B. L., MorRIs, R. G. M. (1987). Hippocampal synaptic enhancement and information storage within a distributed memory system. Trends in Neurosciences, 10, 408-415.
Milner, B. S., Corin, S., \& Teuber, H.-L. (1968). Further analysis of the hippocampal amnesic syndrome: 14-year followup study of H.M. Neuropsychologia, 6, 215-234.

MiYashitA, Y., \& ChaNG, H. S. (1988). Neuronal correlate of pictorial short-term memory in the primate temporal cortex. Nature, 331, 68-70.

Morris, R. G. M., Garrud, P., Rawlins, J. N. P., \& O'Keefe, J. (1982). Place navigation impaired in rats with hippocampal lesions. Nature, 297, 681-683.

Muller, R. U., Kubie, J. L. (1987). The effects of changes in the environment on the spatial firing of hippocampal complex-spike cells. Journal of Neuroscience, 7, 1951-1968.

Muller, R. U., KubIe, J. L., RANCK, J. B., JR. (1987). Spatial firing patterns of hippocampal complex-spike cells in a fixed environment. Journal of Neuroscience, 7, 1935-1950.

O'KeEFE, J. (1976). Place units in the hippocampus of the freely-moving rat. Experimental Neurology, 51, 78-109.

O'KeEFe, J., \& ConWay, D. H. (1978). Hippocampal place units in the freely-moving rat: Why they fire where they fire. Experimental Brain Research, 31, 573-590.

O'KeEFE, J., \&ostrovsky, J. (1971). The hippocampus as a spatial map: Preliminary evidence from unit activity in the freely moving rat. Brain Research, 34, 171-175.

OKEEFE, J., \& NADEL, L. (1978). The hippocampus as a cognitive map. New York: Oxford University Press.

O’Keefe, J., Nadel, L., Keightly, S., \& Kill, D. (1975). Fornix lesions selectively abolish place learning in the rat. Experimental Neurology, 48, 152-166.

O'KeEFE, J., \& SPEAKman, A. (1987). Single unit activity in the rat hippocampus during a spatial memory task. Experimental Brain Research, 68, 1-27.

Olton, D. S., Walker, J. W., \& Gage, F. H. (1978). Hippocampal connections and spatial discrimination. Brain Research, 139, 295-308.

Quirk, G. J., Muller, R. U., Kubie, J. L., \& RANCK, J. B., JR. (1987). Hippocampal and entorhinal place cells: What happened when the lights went out. Society for Neuroscience Abstracts, 13, 1331.

Ramon y CaJal, S. (1911). Histologie du système nerveux de l'homme et des vertèbres (Vol. 2). Paris: Maloine.

RANCK, J. B., JR. (1973). Studies on single neurons in hippocampal formation and septum in unrestrained rats: Part I. Behavioral correlates and firing repertoires. Experimental Neurology, 41, 461-555.

RolLs, E. T. (1989). The representation and storage of information in neural networks in the primate cerebral cortex and hippocampus. In R. Durbin, C. Maill, \& G. Mitchison (Eds.), The computing neuron (pp. 125-159). Workingham, U.K.: Addison-Wesley.

RUMelharT, D. E., ZIPSER, D. (1986). Feature discovery by competitive learning. In D. E. Rumelhart, J. McClelland, \& The PDP Research Group (Eds.), Parallel distributed processing: Explorations in the microstructure of cognition. Vol. 1. Foundations. (pp. 151-193). Cambridge, MA: MIT Press.

Ruthrich, H., Matthies, H., \& OTt, T. (1982). Long-term changes in synaptic excitability of hippocampal cell populations as a result of training. In C. A. Marsan \& H. Matthies (Eds.), Neuronal plasticiry and memory formation (pp. 589-594). New York: Raven Press.

SCOville, W. B., MiLner, B. (1957). Loss of recent memory after bilateral hippocampal lesions. Journal of Neurology, Neurosurgery \& Psychiatry, 20, 11-12.

ShaRP, P. E. (1989). Computer simulation of hippocampal place cells. Society for Neuroscience Abstracts, 15, 403.

Sharp, P. E., Barnes, C. A., \& McNaughton, B. L. (1987). Effects of aging on environmental modulation of hippocampal evoked responses. Behavioral Neuroscience, 101, 170-178.

SharP, P. E., KubIE, J. L., \& MUller, R. U. (1990). Firing properties of hippocampal neurons in a visually-symmetrical environment: Contributions of multiple sensory cues and mnemonic processes. Journal of Neuroscience, 10, 3093-3105.

Sharp, P. E., McNaughton, B. L., \& Barnes, C. A. (1985). Enhancement of hippocampal field potentials in rats exposed to a novel, complex environment. Brain Research, 339, 361-365.

Sharp, P. E., McNaughton, B. L., \& Barnes, C. A. (1989). 
Exploration-dependent modulation of evoked responses in fascia dentata: Fundamental observations and time course. Psychobiology, 17, 257-269.

Skaggs, W. E., \& McNaughton, B. L. (1989). A simple model for hippocampal place cells in which directional tuning of place fields depends on environmental complexity. Society for Neuroscience $A b$ stracts, 15, 776.

Skelton, R. W., Scarth, A. S., Wilkie, D. M., Miller, J. J., \& PHILIPs, A. G. (1987). Long-term increases in dentate granule cell responsivity accompany operant conditioning. Journal of Neuroscience, 7, 3081-3087.

Sutherland, R. J., \& RUDY, J. W. (1989). Configural association theory: The role of the hippocampal formation in learning, memory, and amnesia. Psychobiology, 17, 129-144.

Sutherland, R. J., Whishaw, I. Q., \&olb, B. (1983). A behavioral analysis of spatial localization following electrolytic, kainate- or colchicine-induced damage. Behavioral Brain Research, 7, 133-153.

Van Hoesen, G. W., Pandya, D. N. (1975). Some connections of the entorhinal area (area 28) and perirhinal area (area 35) cortices in the monkey: I. Temporal lobe afferents. Brain Research, 95, 1-24.
Van Hoesen, G. W., Pandya, D. N., Butters, N. (1975). Some connections of the entorhinal area (area 28) and perirhinal area (area 35) cortices in the monkey: II. Frontal lobe afferents. Brain Research, 95, 25-38.

VANDERWOLF, C. H. (1969). Hippocampal electrical activity and voluntary movement in the rat. Electroencephalography \& Clinical Neurophysiology, 26, 407-418.

VON DER MALSBURG, C. (1973). Self-organizing of orientation sensitive cells in the striate cortex. Kybernetik, 14, 85-100.

WeIsz, D. J. (1982). Activity of the dentate gyrus during nictitating membrane conditioning in the rabbit. In C. D. Woody (Ed.), Conditioning: Representations of involved neural functions. Advances in behavioral biology (pp. 131-146). New York: Plenum.

ZIPSER, D. (1985). A computational model of hippocampal place fields. Behavioral Neuroscience, 99, 1006-1018.

(Manuscript received December 19, 1990; revision accepted for publication April 8, 1991.) 